\title{
A Political Ecology of Value: A Cohort-Based Ethnography of the Environmental Turn in Nicaraguan Urban Social Policy
}

\author{
Josh Fisher ${ }^{\ddagger}$, Alex M Nading $§$ \\ ‡ High Point University, High Point, NC, United States of America \\ $\S$ University of Edinburgh, Edinburgh, United Kingdom
}

Corresponding author: Josh Fisher (ifisher@highpoint.edu)

Reviewable v1

Received: 04 Apr 2016| Published: 05 Apr 2016

Citation: Fisher J, Nading A (2016) A Political Ecology of Value: A Cohort-Based Ethnography of the

Environmental Turn in Nicaraguan Urban Social Policy. Research Ideas and Outcomes 2: e8720. doi: 10.3897/ rio.2.e8720

\section{Abstract}

\section{Background}

Research in the social sciences has shown that effective urban policy must strike a delicate balance between social, economic, and environmental considerations. In a world where over half of the world's population now lives in cities, finding this balance is crucial because poor urban planning and management may have costly consequences in economic growth, public health, general well-being, and environmental quality. In recent years, a number of American cities have exhibited the effects of infrastructural failures: flagging economic investment, social unrest, and disease outbreaks. But while the results of are clear, the solutions are not. In the research supported by this award, anthropologists Dr. Joshua B. Fisher (High Point University) and Dr. Alex Nading (University of Edinburgh) will address the problem through innovative research in an urban context whose history of experimentation with policy alternatives and relatively small size, will be particularly revealing of what works, what does not, for whom, and why. 


\section{New information}

The researchers will travel to Ciudad Sandino, a section of peri-urban Managua, Nicaragua. Recently, in response to increased income inequality, urban environmental degradation, and declining revenues from tourism and foreign investment, the city has undertaken a new, cross-sector, integrated urban development campaign, "Live Clean, Live Healthy, Live Beautiful, Live Well." The researchers will examine the complex impacts of this program upon matters of waste management, environmental education, food safety, and public health. They will collect data with a suite of ethnographic research methods including ethnographic interviews, participant observation, photo documentation, and archival analysis. In addition to these more traditional research methods, the researchers will also collect data interactively and dynamically by creating and following a longitudinal ethnographic cohort. Through a series of workshops over three years, a diverse array of citizens including policy-makers, teachers, and informal economy food producers and garbage pickers will be asked to provide feedback to each other and to the researchers on how the programs are affecting them and their worlds. Findings from this research will provide insight into the factors that shape the development of effective urban social policy and successful cities, given their many entangled social, economic, and environmental dimensions.

\section{Keywords}

urban political ecology, urban anthropology, collaborative anthropology, economic anthropology, environmental anthropology, medical anthropology, value

\section{Roles and responsibilities}

There are four principle areas of responsibility:

- $\quad$ management of the data management plan (DMP);

- training other investigators and non-project staff in data collection and/or management in the field;

- short-term storage and backup of the data during and immediately following the data collection period;

- dissemination of data during and immediately following the data collection period;

- long-term storage and custodianship of the data.

The project PI Josh Fisher and project Co-PI Alex Nading will be responsible for the monitoring and administration of the data management plan (DMP); data collection and training other investigators and non-project staff in data collection and/or management in the field; administering and monitoring the short-term storage and backup of the data; and disseminating certain parts of the data (workshop materials, photos, videos) to research participants during the data collection period. 
Three years after the data collection period, the PI and Co-PI will transfer long-term custodianship of the data in perpetuity to the National Anthropological Archives (NAA) at the Smithsonian Institute in Washington, DC. The American Anthropological Association (AAA) Data Registry Wiki will provide additional access to the data.

\section{Expected data}

The research will produce the following types of data in accordance with IRB Human Subjects Protocol:

Texts, including:

- fieldnotes recorded by investigators during workshops and participant observation activities that concern the organization and implementation of the "Live Clean, Live Healthy" campaign as well as the daily activities of workshop participants;

- $\quad$ anonymized text transcriptions of interviews, carried out in Spanish, that concern the research participants' daily activities, observations about Managua's changing environment, and the effects of new urban social policies;

- anonymized text transcriptions of longitudinal ethnographic cohort workshops, carried out in Spanish, that concern themes such as the impact of the "Live Clean, Live Healthy" campaign on forms of citizenship, environmental education, urban farming, recycling and waste scavenging, and large-scale environmental transformations such as the pending transoceanic canal;

- digital photographs and video recordings of public work activities and events associated with the "Live Clean, Live Healthy" campaign or the transoceanic canal;

- $\quad$ archives of news reports, government documents, and other articles pertaining to the history, implementation, and activities of the "Live Clean, Live Healthy" campaign, the construction of the transoceanic canal, or the urban environment in Managua, Nicaragua.

\section{Period of data retention}

The $\mathrm{PI}$ and Co-PI will retain exclusive right to use the data for three years following the conclusion of the data collection period in order to complete coding, analysis, and publication of results, including several single author and co-authored journal articles and a co-authored book. Certain elements of the data set, including transcriptions of workshop materials, digital photographs, and other media, will be made available to research participants during the data collection period. This is because our methodology emphasizes ongoing dialogue with research participants about research methods and tentative conclusions.

Three years after the data collection period concludes, the data will be made publicly available in perpetuity through the National Anthropological Archives (NAA) at the 
Smithsonian Institute and searchable on the American Anthropological Association (AAA) Data Registry Wiki.

\section{Data format and dissemination}

The PI and Co-PI plan to disseminate data from the project to several different specialist and non-specialist audiences. During the data collection process, certain elements of the data (including workshop materials, photos, and videos) will be disseminated to research participants in order to facilitate open dialogue about methodologies and tentative conclusions. In the longer term, data will be transferred to NAA for long-term storage and dissemination. Consequently, all data will be stored in a digital medium that will simultaneously allow for maximum accessibility as well as longevity of storage.

Texts of investigator fieldnotes will remain in their original language and will be saved in a non-proprietary form such as Rich Text Format (.rtf) in order to maximize accessibility on multiple software platforms. In accordance with IRB protocol, this textual data will be anonymized and will not be made available to workshop participants during the data collection period.

Texts of workshop materials, which include research questions and tentative conclusions, will also remain in their original language and will be saved in a non-proprietary form such as Rich Text Format (.rtf) in order to maximize accessibility on multiple software platforms. They will be made available to workshop participants in this format and, when requested, in printed form.

Audio recordings of all interviews and workshops will be transcribed into textual form and anonymized in accordance with IRB Human Subjects protocol regarding protection of confidentiality. They will also remain in their original language and will be saved in a nonproprietary form such as RTF.

Digital photographs of public will be retained as JPEG (.jpg) if originally created in that format. Otherwise, files will be converted and retained in TIFF version 6 uncompressed (.tif) formats. Geospatial metadata, if applicable, will be retained with geo-referenced TIFF (.tif) files. They will be made available to workshop participants in this format and, when requested, in printed form.

Video recordings will be retained in MPEG-4 (.mp4) format. Geospatial metadata, if applicable, will be retained with geo-referenced MPEG-4 (.mp4) files. They will be made available to workshop participants in this format.

Archival materials of news reports, government documents, and other pertinent articles will be scanned in color and at maximum available resolution. They will then be saved as Portable Document Format (.pdf) in order to retain original formatting and to capture nontextual media. 


\section{Data storage and preservation of access}

During the data collection period and in the three subsequent years, all data will be stored in digital form on a secure university server as well as backed up on a third-party server such as Dropbox.

Three years subsequent to the conclusion of the data collection period, custodianship of the data will be transferred in digital form to the National Anthropology Archives (NAA). The NAA retains data on government servers in electronic format as well as in printed form at the Smithsonian Institute in Washington, DC. The NAA provides cost-free electronic access to fieldnotes, transcriptions, images, and videos through the Smithsonian Onling Catalog (SIRIS). Videos are also accessible throught he Human Studies Film Archives (HSFA).

\section{Additional possible data management requirements}

The data will also be listed in the American Anthropological Association (AAA) Data Registry Wiki, funded by the American Anthropological Association (AAA) and the National Science Foundation grant BCS-1159109. This listing has numerous advantages, including acceleration of data sharing, citation, and further discovery.

\section{Project}

National Science Foundation (NSF) Social Behavioral and Economic Sciences (SBE) Template

Public Data Management Plan created with the DMPTool: https://dmptool.org/ plans/19207.pdf 